\title{
Embedding formulae for scattering in a waveguide containing polygonal obstacles
}

Article

Accepted Version

Biggs, N. R. T. (2016) Embedding formulae for scattering in a waveguide containing polygonal obstacles. The Quarterly Journal of Mechanics and Applied Mathematics, 69 (4). pp. 409-429. ISSN 0033-5614 doi:

https://doi.org/10.1093/qjmam/hbw012 Available at https://centaur.reading.ac.uk/66108/

It is advisable to refer to the publisher's version if you intend to cite from the work. See Guidance on citing.

To link to this article DOI: http://dx.doi.org/10.1093/qjmam/hbw012

Publisher: Oxford University Press

All outputs in CentAUR are protected by Intellectual Property Rights law, including copyright law. Copyright and IPR is retained by the creators or other copyright holders. Terms and conditions for use of this material are defined in the End User Agreement.

\section{www.reading.ac.uk/centaur}

\section{CentAUR}

Central Archive at the University of Reading 
Reading's research outputs online 


\title{
EMBEDDING FORMULAE FOR SCATTERING IN A WAVEGUIDE CONTAINING POLYGONAL OBSTACLES
}

\author{
by N.R.T. BIGGS ${ }^{\ddagger}$ \\ (Department of Mathematics and Statistics, School of Mathematical and Physical Sciences, \\ University of Reading, P.O. Box 220, Whiteknights, Reading RG6 7AX, U.K.)
}

[Received ? November 2015. Revise ?]

\begin{abstract}
Summary
For certain wave scattering problems embedding formulae can be derived, which express the solution, or far-field behaviour of the solution, for arbitrary plane wave incident angle in terms of the corresponding quantities for a finite number of other related problems. Their scope has so far been limited to scattering in $\mathbb{R}^{2}$, and to a lesser extent $\mathbb{R}^{3}$; in this paper we derive embedding formulae for wave scattering in a class of two-dimensional waveguide. The waveguide is straight and of uniform width outside a finite length region within which the boundaries are piecewise-linear and the waveguide can contain polygonal obstacles, a restriction being that all boundaries of the waveguide and obstacles must be inclined at a rational angle to the axis of the waveguide. Once solutions are determined for a finite set of incident propagating modes, the embedding formulae provide expressions for reflection and transmission coefficients for all remaining incident propagating modes. The precise number of solutions required is a function of the number and nature of the corners of the boundaries and obstacles. The formulae are illustrated for a particular waveguide geometry for which the problem can be formulated as an integral equation and approximate numerical solutions determined using the Galerkin method.
\end{abstract}

\section{Introduction}

To fully characterise the wave scattering properties of an obstacle, solutions of the scattering problem are typically required for a range of plane wave incident angles. One useful means of minimising the effort required to achieve this full characterisation is offered by embedding formulae, which express the solution for any incident plane wave angle in terms of solutions corresponding to a finite, usually small, number of other solutions.

The range of scattering geometries for which such formulae have been derived is currently far from extensive. In (1) and (2) it was demonstrated that the classical two-dimensional problem of a plane wave incident upon a single gap in an otherwise infinite, thin, straight, sound-hard barrier admits such a formula: the solution for any incident plane angle can be expressed in terms of just one solution, corresponding to grazing plane wave incidence.

The results of ( 2 and subsequent extensions to multiple gaps (3), thick barriers (4), a perforated duct (5) and non-colinear strips (6), all require that the problems be formulated as an integral equation, and the structure of this equation is then exploited to derive the required results. In each case, solutions for arbitrary incident wave angle are expressed

$\ddagger$ (n.r.t.biggs@reading.ac.uk)

Q. Jl Mech. Appl. Math. (?) ? (?), 1-21

(c) Oxford University Press ? 
in terms of solutions corresponding to other incident plane wave angles. In contrast, the papers (7-10) address the initial governing boundary-value problem directly, and express plane wave solutions in terms of solutions forced by multipole sources positioned at the vertices of the scatterer. In particular, (8) significantly extends the class of scattering geometry for which embedding formulae can be derived to include scatterers whose straight boundaries are inclined at an angle $m \pi / n$, for integer $m, n$, to the $x$-axis. Subsequently, the approach was modified in (11) to allow, for this class of scatterer, embedding formulae in terms of solutions forced by incident plane waves, rather than by multipoles.

Until now, embedding formulae have only been derived for plane wave scattering in $\mathbb{R}^{2}$, and to a lesser extent $\mathbb{R}^{3}$ (in (9)). In the current paper, the method of (8) as adapted in (11) is extended to the case of wave scattering in a two-dimensional waveguide, on the boundaries of which a homogeneous Dirichlet is imposed for simplicity. The restriction on the alignment of the boundaries is as in (8) and (11): taking the centreline of the waveguide aligned with the $x$-axis, its boundaries are allowed to be piecewise linear in profile, and the waveguide can contain obstacles whose boundaries are piecewise linear, provided that each linear portion of the waveguide or obstacle boundary makes an angle with the waveguide axis which is a rational multiple of $\pi$.

Only finitely many propagating waves exist within the waveguide, rather than the continuum of incident plane wave angles for the $\mathbb{R}^{2}$ scattering problems described above. Thus in the current context embedding formulae relate reflection and transmission coefficients of these propagating waves.

The paper proceeds as follows. In section 2 the details of the boundary-value problem to be considered are laid out. In section 3 a straight waveguide containing a single straight, thin barrier, aligned with the waveguide axis, is first considered, since this is the simplest waveguide geometry for which embedding formulae can be derived. The extension to multiple such barriers is then covered, before the general case is examined in section 4 . The calculation of the number of "overly-singular" terms produced at the corners of the waveguide and the obstacles by a particular differential operator proves to be the main task. Some particular waveguide geometries are then discussed, and in section 5 the problem for one such geometry is formulated as an integral equation, solved approximately using Galerkin's method, and the corresponding embedding formulae are implemented. Finally, section 6 summarises and discusses the findings.

\section{The boundary-value problem}

Let $(x, y)$ denote Cartesian coordinates, and let $L>0$ be a constant. In $\mp x>L$ the waveguide occupies the regions $\left\{\mp x>L, a_{ \pm}<y<b_{ \pm}\right\}$, for given constants $a_{ \pm}, b_{ \pm}$. For $-L<x<L$ the waveguide has piecewise linear boundaries, and can also contain obstacles whose boundaries are piecewise linear. A sketch of a typical waveguide geometry is displayed in Fig. 1. More detail on the possible form of the boundaries and the obstacles is given below.

We consider solutions of the reduced wave equation

$$
u_{x x}+u_{y y}+\kappa^{2} u=0
$$

where $\kappa$ is the prescribed wavenumber, and suppose that $u$ satisfies a homogeneous Dirichlet condition $u=0$ on the waveguide walls; only minor modifications are required if $u$ instead 


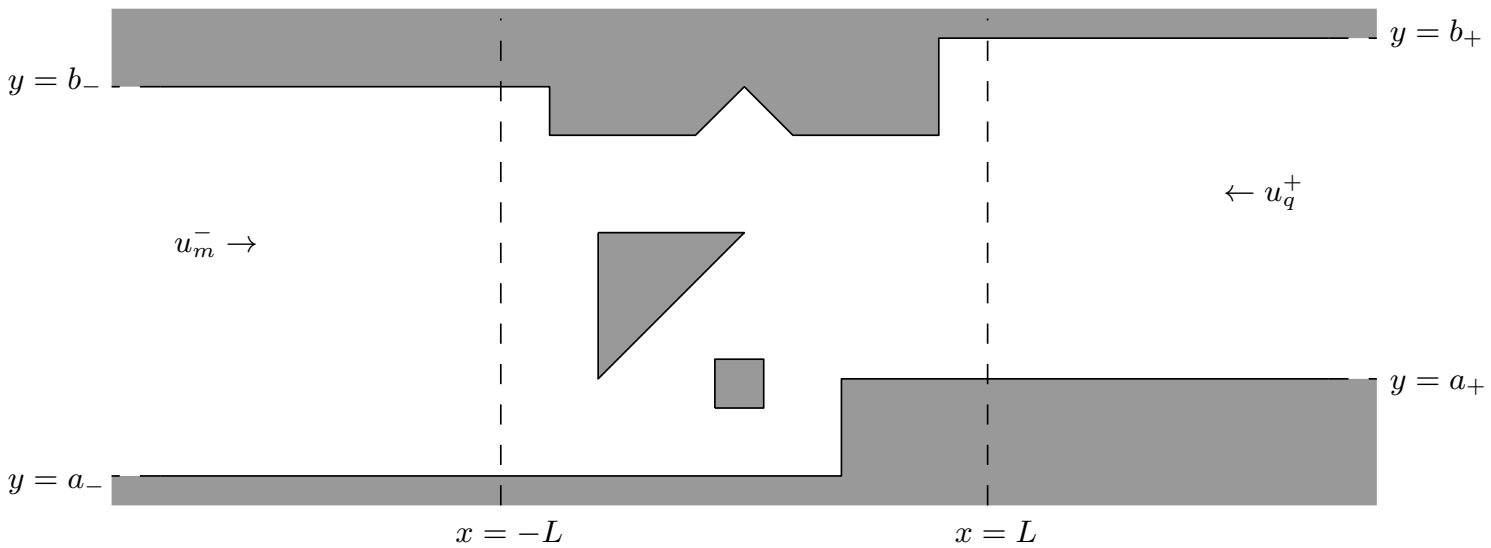

Fig. 1 Example of waveguide geometry.

satisfies a Neumann condition. Near a corner in the waveguide for which, in terms of polar coordinates $(r, \theta)$ centred on the corner, the interval $\theta_{1}<\theta<\theta_{2}$ is inside the guide, the solution must behave as

$$
u=O\left(r^{\nu}\right), \quad \nu=\pi /\left(\theta_{2}-\theta_{1}\right),
$$

the so-called Meixner condition. The solution must also satisfy appropriate radiation conditions as $x \rightarrow \pm \infty$, which are specified below.

The general solution as $x \rightarrow \pm \infty$ is of the form

$$
u(x, y) \sim \sum_{n=1}^{N_{ \pm}}\left(c_{n}^{ \pm} e^{\mp i \kappa_{n}^{ \pm} x}+d_{n}^{ \pm} e^{ \pm i \kappa_{n}^{ \pm} x}\right) Y_{n}^{ \pm}(y)
$$

for constants $c_{n}^{ \pm}, d_{n}^{ \pm}$, where the functions

$$
Y_{n}^{ \pm}(y)=\sqrt{2 /\left(b_{ \pm}-a_{ \pm}\right)} \sin \left[\alpha_{n}^{ \pm}\left(y-a_{ \pm}\right)\right], \quad \alpha_{n}^{ \pm}=n \pi /\left(b_{ \pm}-a_{ \pm}\right) \quad(n \in \mathbb{N})
$$

are orthonormal on $\left(a_{ \pm}, b_{ \pm}\right)$, and

$$
\kappa_{n}^{ \pm}= \begin{cases}\sqrt{\kappa^{2}-\alpha_{n}^{ \pm}} & \left(\kappa \geq \alpha_{n}^{ \pm}\right), \\ i \sqrt{\alpha_{n}^{ \pm}-\kappa^{2}} & \left(\kappa \leq \alpha_{n}^{ \pm}\right) .\end{cases}
$$

Here $N_{ \pm}=\left[\kappa\left(b_{ \pm}-a_{ \pm}\right) / \pi\right]$, in which [.] denotes the integer part; for convenience we introduce the sets $\bar{N}_{ \pm}=\left\{1, \ldots, N_{ \pm}\right\}$.

We write the potential forced by the $m$-th incident mode from the left $\left(m \in \bar{N}_{-}\right)$as $u_{m}^{-}$; the radiation condition requires that the remainder after subtracting the incident wave from $u_{m}^{-}$must be outgoing and bounded as $x \rightarrow \pm \infty$. As such, $u_{m}^{-}$satisfies

$$
u_{m}^{-}(x, y) \sim \begin{cases}A_{m}^{-} e^{i \kappa_{m}^{-} x} Y_{m}^{-}(y)+A_{m}^{-} \sum_{n=1}^{N_{-}} R_{n m}^{-} e^{-i \kappa_{n}^{-} x} Y_{n}^{-}(y) & (x \rightarrow-\infty) \\ A_{m}^{-} \sum_{n=1}^{N_{+}} T_{n m}^{-} e^{i \kappa_{n}^{+} x} Y_{n}^{+}(y) & (x \rightarrow \infty)\end{cases}
$$


where $A_{m}^{-}$is prescribed and the reflection and transmission coefficients, $R_{n m}^{-}$and $T_{n m}^{-}$, are unknown. Similarly, the potential $u_{q}^{+}$is forced by the $q$-th incident mode from the right $\left(q \in \bar{N}_{+}\right)$, and satisfies

$$
u_{q}^{+}(x, y) \sim \begin{cases}A_{q}^{+} \sum_{n=1}^{N_{-}} T_{n q}^{+} e^{-i \kappa_{n}^{-} x} Y_{n}^{-}(y) & (x \rightarrow-\infty) \\ A_{q}^{+} e^{-i \kappa_{q}^{+} x} Y_{q}^{+}(y)+A_{q}^{+} \sum_{n=1}^{N_{+}} R_{n q}^{+} e^{i \kappa_{n}^{+} x} Y_{n}^{+}(y) & (x \rightarrow \infty),\end{cases}
$$

where $A_{q}^{+}$is prescribed and the reflection and transmission coefficients $R_{n q}^{+}$and $T_{n q}^{+}$, are unknown.

A variety of reciprocity relations link the reflection/transmission coefficients $R_{n m}^{ \pm}, T_{n m}^{ \pm}$. Use of Green's identity shows that if $u, v$ are any solutions of the Helmholtz equation plus boundary conditions in the waveguide, then for $l>L$,

$$
\int_{a_{-}}^{b_{-}}\left\{-u(-l, y) v_{x}(-l, y)+v(-l, y) u_{x}(-l, y)\right\} \mathrm{d} y+\int_{a_{+}}^{b_{+}}\left\{u(l, y) v_{x}(l, y)-v(l, y) u_{x}(l, y)\right\} \mathrm{d} y=0 .
$$

In particular, if $l$ is sufficiently large for the far-field behaviours (2.4) and (2.5) to be valid, then setting $u=u_{m}^{ \pm}$and $v=u_{q}^{ \pm}$, for $m, q \in \bar{N}_{ \pm}$, shows that

$$
\kappa_{m}^{ \pm} R_{m q}^{ \pm}=\kappa_{q}^{ \pm} R_{q m}^{ \pm} \quad\left(m, q \in \bar{N}_{ \pm}\right),
$$

whilst setting $u=u_{m}^{\mp}\left(m \in \bar{N}_{\mp}\right)$ and $v=u_{q}^{ \pm}\left(q \in \bar{N}_{ \pm}\right)$gives

$$
\kappa_{m}^{\mp} T_{m q}^{ \pm}=\kappa_{q}^{ \pm} T_{q m}^{\mp} \quad\left(m \in \bar{N}_{\mp}, q \in \bar{N}_{ \pm}\right) .
$$

\section{Embedding for a straight waveguide containing thin barriers parallel to the $x$-axis}

3(a) A single barrier

To illustrate the process we first consider the simplest situation for which embedding formulae exist, and the simplest such embedding formula which can be derived. Suppose that the waveguide is straight, with $a_{-}=a_{+}$and $b_{-}=b_{+}$, so that in particular $\kappa_{n}^{-} \equiv \kappa_{n}^{+}$, $Y_{n}^{-} \equiv Y_{n}^{+}$and $N_{-} \equiv N_{+}$; the notation $\kappa_{n}^{ \pm}$etc. is retained however, to aid understanding, and assist later extension to cases for which $a_{-}, b_{-} \neq a_{+}, b_{+}$. Suppose further that the waveguide contains a single thin barrier occupying the region $S=\left\{x_{1}<x<x_{2}, y=y_{0}\right\}$, where $a<y_{0}<b$, on which a Dirichlet boundary condition must be satisfied. The Meixner condition (2.2) takes the form $u=O\left(r^{1 / 2}\right)$ near either corner.

The method follows closely the adaptation of (8) given in (11). We introduce the differential operator

$$
H_{m}^{-}=\partial_{x}-i \kappa_{m}^{-} I
$$

for $m \in \bar{N}_{-}$, in terms of which the combination $H_{m}^{-} u_{m}^{-}$satisfies the Helmholtz equation in the guide, the boundary conditions on the waveguide walls and the barrier $S$, contains no incident wave because $H_{m}^{-}\left(e^{i \kappa_{m}^{-} x} Y_{m}^{-}(y)\right)=0$, and is bounded and outgoing as $x \rightarrow \pm \infty$. 
The Meixner conditions at the corners of $S$ are violated however: near $\left(x_{1}, y_{0}\right)$ the solution is of the form

$$
u_{m}^{-}=B_{1, m}^{-} r^{1 / 2} \sin (\theta / 2)+O\left(r^{3 / 2}\right),
$$

where $(r, \theta)$ are polar coordinates centred on $\left(x_{1}, y_{0}\right)$, and $B_{1, m}^{-}$is a constant, but then

$$
H_{m}^{-} u_{m}^{-}=-\frac{1}{2} B_{1, m}^{-} r^{-1 / 2} \sin (\theta / 2)+O\left(r^{1 / 2}\right)
$$

and the $O\left(r^{-1 / 2}\right)$ term is an overly-singular term which contravenes the Meixner condition. Clearly the combination $H_{m}^{-} u_{m}^{-}$also includes a term which is singular at the other end, $\left(x_{2}, y_{0}\right)$, of the scatterer, so we introduce the combination

$$
U=H_{m}^{-} u_{m}^{-}-\left(C H_{j}^{-} u_{j}^{-}+D H_{k}^{-} u_{k}^{-}\right)
$$

in which $m, j, k \in \bar{N}_{-}$are distinct, and for which we can choose the constants $C$ and $D$ so that the coefficients of the two overly-singular terms are zero.

This combination $U$ then satisfies a fully homogeneous problem, but, in contrast to earlier work considering scattering of plane waves by obstacles in $\mathbb{R}^{2}$ for which we could at this stage invoke uniqueness to deduce that $U \equiv 0$, here we cannot necessarily infer this since one or more trapped modes may exist. These are solutions of the homogeneous problem which (crucially for our purposes) decay to zero as $x \rightarrow \pm \infty$, and if they exist for a particular geometry typically do so only at isolated frequencies. Within the class of waveguide geometries considered in this paper their existence can be caused by either the presence of obstacles in the guide (see, e.g. (12)), or by variation in the guide width (see, e.g. (13)).

Thus we can only deduce that $U=\hat{U}$, where here and throughout this paper $\hat{U}$ denotes a linear combination of all trapped modes which exist for the particular geometry and frequency being considered; thus

$$
H_{m}^{-} u_{m}^{-}=C H_{j}^{-} u_{j}^{-}+D H_{k}^{-} u_{k}^{-}+\hat{U} .
$$

Now take the limit of (3.5) as $x \rightarrow-\infty$. Use of the limiting behaviour $\hat{U} \rightarrow 0$, plus the far-field form (2.4), shows that

$$
\begin{aligned}
A_{m}^{-} \sum_{n=1}^{N_{-}}\left(\kappa_{n}^{-}+\kappa_{m}^{-}\right) R_{n m}^{-} e^{-i \kappa_{n}^{-} x} Y_{n}^{-}(y)= & C A_{j}^{-} \sum_{n=1}^{N_{-}}\left(\kappa_{n}^{-}+\kappa_{j}^{-}\right) R_{n j}^{-} e^{-i \kappa_{n}^{-} x} Y_{n}^{-}(y) \\
& +D A_{k}^{-} \sum_{n=1}^{N_{-}}\left(\kappa_{n}^{-}+\kappa_{k}^{-}\right) R_{n k}^{-} e^{-i \kappa_{n}^{-} x} Y_{n}^{-}(y)
\end{aligned}
$$

and then equating coefficients of the modes $e^{-i \kappa_{n}^{-} x} Y_{n}^{-}(y)$ shows that

$$
A_{m}^{-}\left(\kappa_{n}^{-}+\kappa_{m}^{-}\right) R_{n m}^{-}=C A_{j}^{-}\left(\kappa_{n}^{-}+\kappa_{j}^{-}\right) R_{n j}^{-}+D A_{k}^{-}\left(\kappa_{n}^{-}+\kappa_{k}^{-}\right) R_{n k}^{-},
$$

for $n \in \bar{N}_{-}$. 
To determine the constants $C$ and $D$ we set $n=j$ and $n=k$ in (3.6) in turn, and invoke the reciprocity relation $(2.7)$, to give

$$
2 C A_{j}^{-} \kappa_{j}^{-} R_{j j}^{-}+D A_{k}^{-}\left(\kappa_{j}^{-}+\kappa_{k}^{-}\right) R_{j k}^{-}=A_{m}^{-}\left(\kappa_{j}^{-}+\kappa_{m}^{-}\right) R_{j m}^{-} \equiv A_{m}^{-}\left(\kappa_{j}^{-}+\kappa_{m}^{-}\right)\left(\kappa_{m}^{-} / \kappa_{j}^{-}\right) R_{m j}^{-}
$$

and

$$
C A_{j}^{-}\left(\kappa_{k}^{-}+\kappa_{j}^{-}\right) R_{k j}^{-}+2 D A_{k}^{-} \kappa_{k}^{-} R_{k k}^{-}=A_{m}^{-}\left(\kappa_{k}^{-}+\kappa_{m}^{-}\right) R_{k m}^{-} \equiv A_{m}^{-}\left(\kappa_{k}^{-}+\kappa_{m}^{-}\right)\left(\kappa_{m}^{-} / \kappa_{k}^{-}\right) R_{m k}^{-} .
$$

These two equations can now be solved to give

$$
C=\frac{A_{m}^{-}\left(\tilde{R}_{k k}^{-} \tilde{R}_{m j}^{-}-\tilde{R}_{j k}^{-} \tilde{R}_{m k}^{-}\right)}{A_{j}^{-}\left(\tilde{R}_{j j}^{-} \tilde{R}_{k k}^{-}-\tilde{R}_{j k}^{-} \tilde{R}_{k j}^{-}\right)}, \quad D=\frac{A_{m}^{-}\left(\tilde{R}_{j j}^{-} \tilde{R}_{m k}^{-}-\tilde{R}_{j k}^{-} \tilde{R}_{m j}^{-}\right)}{A_{k}^{-}\left(\tilde{R}_{j j}^{-} \tilde{R}_{k k}^{-}-\tilde{R}_{j k}^{-} \tilde{R}_{k j}^{-}\right)}
$$

in which for convenience we've introduced the scaled reflection coefficients

$$
\tilde{R}_{m j}^{ \pm}=\kappa_{m}^{ \pm}\left(\kappa_{m}^{ \pm}+\kappa_{j}^{ \pm}\right) R_{m j}^{ \pm} \quad\left(m, j \in \bar{N}_{ \pm}\right)
$$

(and in terms of which the reciprocity relation (2.7) is simply $\tilde{R}_{m j}^{ \pm}=\tilde{R}_{j m}^{ \pm}$). Inserting these expressions back into (3.6) then results in the equality

$$
\left(\tilde{R}_{j j}^{-} \tilde{R}_{k k}^{-}-\tilde{R}_{j k}^{-} \tilde{R}_{k j}^{-}\right) \tilde{R}_{n m}^{-}=\left(\tilde{R}_{k k}^{-} \tilde{R}_{m j}^{-}-\tilde{R}_{j k}^{-} \tilde{R}_{m k}^{-}\right) \tilde{R}_{n j}^{-}-\left(\tilde{R}_{k j}^{-} \tilde{R}_{m j}^{-}-\tilde{R}_{j j}^{-} \tilde{R}_{m k}^{-}\right) \tilde{R}_{n k}^{-} .
$$

Equation (3.9) is an embedding formula, since once the solutions $u_{j}^{-}$and $u_{k}^{-}$are determined, for any distinct $j, k \in \bar{N}_{-}$, and in particular their behaviour as $x \rightarrow-\infty$ is known, (3.9) can be used to construct all of the remaining reflection coefficients $R_{n m}^{-}$for $n, m \in \bar{N}_{-}$.

\section{3(b) Multiple barriers}

Suppose now that there are $M^{\prime} \geq 1$ thin straight barriers in the straight guide, all parallel to the $x$-axis but otherwise in any arrangement. The combination $H_{m}^{-} u_{m}^{-}$now includes $M=2 M^{\prime}$ overly-singular terms, one for each barrier tip.

We generalise the method of section 3(a), and derive an embedding formula which expresses the scattering coefficients for the solution $u_{m}^{-}$in terms of scattering coefficients for a combination of $M$ solutions forced by waves incident from both $+\infty$ and $-\infty$. (Note we are assuming here that the total number of possible incident waves, $N_{+}+N_{-}$, is less than $M$, so that $M$ distinct integers can be picked from $\bar{N}_{-} \cup \bar{N}_{+}$. This condition will be most restrictive at low frequencies.) Thus we pick distinct integers $l_{1}^{-}, l_{2}^{-}, \ldots, l_{M_{-}}^{-}$in $\bar{N}_{-}$ and $l_{1}^{+}, l_{2}^{+}, \ldots, l_{M_{+}}^{+}$in $\bar{N}_{+}$, where the integers $M_{ \pm} \geq 0$ are such that $M_{-}+M_{+}=M$, and also extend (3.1) to

$$
H_{m}^{ \pm}=\partial_{x} \pm i \kappa_{m}^{ \pm} I \quad\left(m \in \bar{N}_{ \pm}\right)
$$

The analogue of $(3.4)$ is

$$
U=H_{m}^{-} u_{m}^{-}-\sum_{j=1}^{M_{-}} C_{j}^{-} H_{l_{j}^{-}}^{-} u_{l_{j}^{-}}^{-}-\sum_{j=1}^{M_{+}} C_{j}^{+} H_{l_{j}^{+}}^{+} u_{l_{j}^{+}}^{+},
$$

in which the coefficients $C_{j}^{ \pm}$can be chosen so that the Meixner conditions are satisfied at 
each corner. The combination $U$ satisfies a fully homogeneous problem, from which it is then immediate that

$$
H_{m}^{-} u_{m}^{-}=\sum_{j=1}^{M_{-}} C_{j}^{-} H_{l_{j}^{-}}^{-} u_{l_{j}^{-}}^{-}+\sum_{j=1}^{M_{+}} C_{j}^{+} H_{l_{j}^{+}}^{+} u_{l_{j}^{+}}^{+}+\hat{U} .
$$

Now take the limit $x \rightarrow-\infty$ in (3.12), use the far-field form (2.4) and the limiting behaviour $\hat{U} \rightarrow 0$, and finally equate coefficients of $e^{-i \kappa_{n}^{-} x} Y_{n}^{-}(y)$. The result is

$$
A_{m}^{-}\left(\kappa_{n}^{-}+\kappa_{m}^{-}\right) R_{n m}^{-}=\sum_{j=1}^{M_{-}} C_{j}^{-} A_{l_{j}^{-}}^{-}\left(\kappa_{n}^{-}+\kappa_{l_{j}^{-}}^{-}\right) R_{n, l_{j}^{-}}^{-}+\sum_{j=1}^{M_{+}} C_{j}^{+} A_{l_{j}^{+}}^{+}\left(\kappa_{n}^{-}-\kappa_{l_{j}^{+}}^{+}\right) T_{n, l_{j}^{+}}^{+}
$$

for $n \in \bar{N}_{-}$. In particular, setting $n=l_{k}^{-}\left(k=1,2, \ldots, M_{-}\right)$in (3.13) gives

$$
\begin{aligned}
\sum_{j=1}^{M_{-}} C_{j}^{-} A_{l_{j}^{-}}^{-}\left(\kappa_{l_{k}^{-}}^{-}+\kappa_{l_{j}^{-}}^{-}\right) R_{l_{k}^{-}, l_{j}^{-}}^{-}+\sum_{j=1}^{M_{+}} C_{j}^{+} A_{l_{j}^{+}}^{+}\left(\kappa_{l_{k}^{-}}^{-}-\kappa_{l_{j}^{+}}^{+}\right) T_{l_{k}^{-}, l_{j}^{+}}^{+} & =A_{m}^{-}\left(\kappa_{l_{k}^{-}}^{-}+\kappa_{m}^{-}\right) R_{l_{k}^{-}, m}^{-} \\
& \equiv A_{m}^{-}\left(\kappa_{l_{k}^{-}}^{-}+\kappa_{m}^{-}\right) \frac{\kappa_{m}^{-}}{\kappa_{l_{k}^{-}}^{-}} R_{m, l_{k}^{-}}^{-}
\end{aligned}
$$

in which the final equality follows after use of the reciprocity relation (2.7). In terms of the notation introduced in (3.8), together with the addition of

$$
\tilde{T}_{m j}^{ \pm}=\kappa_{m}^{\mp}\left(\kappa_{m}^{\mp}-\kappa_{j}^{ \pm}\right) T_{m j}^{ \pm}
$$

(in terms of which the reciprocity relation $(2.8)$ is $\tilde{T}_{m j}^{ \pm}=-\tilde{T}_{j m}^{\mp}$ ) equation (3.14) can be written more succinctly as

$$
\sum_{j=1}^{M_{-}} C_{j}^{-} A_{l_{j}^{-}}^{-} \tilde{R}_{l_{k}^{-}, l_{j}^{-}}^{-}+\sum_{j=1}^{M_{+}} C_{j}^{+} A_{l_{j}^{+}}^{+} \tilde{T}_{l_{k}^{-}, l_{j}^{+}}^{+}=A_{m}^{-} \tilde{R}_{m, l_{k}^{-}}^{-} \quad\left(k=1,2, \ldots, M_{-}\right) .
$$

Equation (3.16) constitutes $M_{-}$equations from which to determine the $M_{-}+M_{+}$ unknowns $C_{j}^{ \pm}$; the remaining $M_{+}$equations are found through taking the limit of (3.12) as $x \rightarrow \infty$ and equating coefficients of $e^{i \kappa_{n}^{+} x} Y_{n}^{+}(y)$, to yield

$$
A_{m}^{-}\left(\kappa_{n}^{+}-\kappa_{m}^{-}\right) T_{n m}^{-}=\sum_{j=1}^{M_{-}} C_{j}^{-} A_{l_{j}^{-}}^{-}\left(\kappa_{n}^{+}-\kappa_{l_{j}^{-}}^{-}\right) T_{n, l_{j}^{-}}^{-}+\sum_{j=1}^{M_{+}} C_{j}^{+} A_{l_{j}^{+}}^{+}\left(\kappa_{n}^{+}+\kappa_{l_{j}^{+}}^{+}\right) R_{n, l_{j}^{+}}^{+},
$$

for $n \in \bar{N}_{+}$. Now setting $n=l_{k}^{+}\left(k=1,2, \ldots, M_{+}\right)$in (3.17) gives

$$
\begin{aligned}
\sum_{j=1}^{M_{-}} C_{j}^{-} A_{l_{j}^{-}}^{-}\left(\kappa_{l_{k}^{+}}^{+}-\kappa_{l_{j}^{-}}^{-}\right) T_{l_{k}^{+}, l_{j}^{-}}^{-}+\sum_{j=1}^{M_{+}} C_{j}^{+} A_{l_{j}^{+}}^{+}\left(\kappa_{l_{k}^{+}}^{+}+\kappa_{l_{j}^{+}}^{+}\right) R_{l_{k}^{+}, l_{j}^{+}}^{+} & =A_{m}^{-}\left(\kappa_{l_{k}^{+}}^{+}-\kappa_{m}^{-}\right) T_{l_{k}^{+}, m}^{-} \\
& \equiv A_{m}^{-}\left(\kappa_{l_{k}^{+}}^{+}-\kappa_{m}^{-}\right) \frac{\kappa_{m}^{-}}{\kappa_{l_{k}^{+}}^{+}} T_{m, l_{k}^{+}}^{+},
\end{aligned}
$$


where the reciprocity relation $(2.8)$ has been used. This can be written in the notation of (3.8) and (3.15) as

$$
\sum_{j=1}^{M_{-}} C_{j}^{-} A_{l_{j}^{-}}^{-} \tilde{T}_{l_{k}^{+}, l_{j}^{-}}^{-}+\sum_{j=1}^{M_{+}} C_{j}^{+} A_{l_{j}^{+}}^{+} \tilde{R}_{l_{k}^{+}, l_{j}^{+}}^{+}=-A_{m}^{-} \tilde{T}_{m, l_{k}^{+}}^{+} \quad\left(k=1,2, \ldots, M_{+}\right) .
$$

Equations (3.16) and (3.19) together constitute $M_{-}+M_{+}=M$ equations from which to determine the $M$ unknowns $C_{j}^{ \pm}$. Inserting these values for the $C_{j}^{ \pm}$into (3.13) yields an embedding formula which expresses the reflection coefficients $R_{n m}^{-}$, for all $n, m \in \bar{N}_{-}$, in terms of quantities which rely only on the solutions $u_{l_{j}^{ \pm}}^{ \pm}, j=1,2, \ldots, M_{ \pm}$. Similarly, inserting these expressions for the $C_{j}^{ \pm}$into (3.17) yields an embedding formula for the transmission coefficients $T_{n m}^{-}$, for all $m \in \bar{N}_{-}, n \in \bar{N}_{+}$.

We note that it is possible to confirm directly that if the constants $C_{j}^{ \pm}$in (3.11) can be chosen to eliminate the overly-singular behaviour at the $M$ barrier corners, i.e. to satisfy

$$
\sum_{j=1}^{M_{-}} B_{k, l_{j}^{-}}^{-} C_{j}^{-}+\sum_{j=1}^{M_{+}} B_{k, l_{j}^{+}}^{+} C_{j}^{+}=B_{k m}^{-} \quad(k=1, \ldots, M),
$$

in which $B_{k j}^{ \pm}$is the coefficient of the leading order term in an expansion of $u_{j}^{ \pm}$near the $k$-th corner (see (3.2)), then the expressions for $C_{j}^{ \pm}$which result coincide with those found from solving (3.16) and (3.19) for the $C_{j}^{ \pm}$in terms of reflection/transmission coefficients. The connection is provided by reciprocity relations similar to (2.7) and (2.8), but in which the overly-singular behaviour of the integrand at the barrier corners produces extra terms (similar cases are considered in e.g. (10)). Thus applying Green's identity to the pairs $u_{n}^{-}$ and $H_{p}^{-} u_{p}^{-}$, and $u_{q}^{-}$and $H_{s}^{p} u_{s}^{p}$, results in the identities

$$
A_{p}^{-}\left(\kappa_{n}^{-}+\kappa_{p}^{-}\right) R_{n p}^{-}=\frac{\pi}{4 \kappa_{n}^{-} A_{n}^{-}} \sum_{l=1}^{M} B_{l n}^{-} B_{l p}^{-}, \quad A_{q}^{+}\left(\kappa_{n}^{-}-\kappa_{q}^{+}\right) T_{n q}^{+}=\frac{\pi}{4 \kappa_{n}^{-} A_{n}^{-}} \sum_{l=1}^{M} B_{l n}^{-} B_{l q}^{+},
$$

for $n, p \in \bar{N}_{-}, q \in \bar{N}_{+}$, with two further identities, for $R_{p n}^{+}$and $T_{q n}^{-}$, forthcoming upon combining (3.21) with (2.7) and (2.8) respectively. Use of these identities then allows (3.16) and (3.19) to be rewritten as

$$
\begin{cases}\sum_{l=1}^{M} B_{l, l_{k}^{-}}^{-}\left(\sum_{j=1}^{M_{-}} B_{l, l_{j}^{-}}^{-} C_{j}^{-}+\sum_{j=1}^{M_{+}} B_{l, l_{j}^{+}}^{+} C_{j}^{+}\right)=\sum_{l=1}^{M} B_{l, l_{k}^{-}}^{-} B_{l m}^{-} \quad\left(k=1, \ldots, M_{-}\right), \\ \sum_{l=1}^{M} B_{l, l_{k}^{+}}^{+}\left(\sum_{j=1}^{M_{-}} B_{l, l_{j}^{-}}^{-} C_{j}^{-}+\sum_{j=1}^{M_{+}} B_{l, l_{j}^{+}}^{+} C_{j}^{+}\right)=\sum_{l=1}^{M} B_{l, l_{k}^{+}}^{+} B_{l m}^{-} \quad\left(k=1, \ldots, M_{+}\right) .\end{cases}
$$

To clarify the relationship between (3.20) and (3.22) we write the former as

$$
B\left(\begin{array}{l}
\mathbf{C}^{-} \\
\mathbf{C}^{+}
\end{array}\right)=\mathbf{B}_{m}^{-},
$$


in which $B=\left(B^{-} B^{+}\right)$and $B^{ \pm}$is $M \times M_{ \pm}$with $(p, q)$-th entry $B_{p, l_{q}^{ \pm}}^{ \pm}, \mathbf{C}^{ \pm}$is $M_{ \pm} \times 1$ with $j$-th entry $C_{j}^{ \pm}$, and $\mathbf{B}_{m}^{-}$is $M \times 1$ with $k$-th entry $B_{k m}^{-}$; the latter can then be identified as

$$
B^{T} B\left(\begin{array}{c}
\mathbf{C}^{-} \\
\mathbf{C}^{+}
\end{array}\right)=B^{T} \mathbf{B}_{m}^{-}
$$

Thus if $\mathbf{C}^{ \pm}$can be determined from (3.23) then in particular $\operatorname{det}(B)$ is non-zero so that (3.24) can be reduced to precisely (3.23), and the solutions of the two systems coincide.

Two particular cases of the embedding formulae are worth writing out in full. If $M_{-}=M$ and $M_{+}=0$ then the $C_{j}^{+}$and equations of (3.19) are omitted, and equations (3.13) and (3.17) become

$$
A_{m}^{-}\left(\kappa_{n}^{-}+\kappa_{m}^{-}\right) R_{n m}^{-}=\sum_{j=1}^{M} C_{j}^{-} A_{l_{j}^{-}}^{-}\left(\kappa_{n}^{-}+\kappa_{l_{j}^{-}}^{-}\right) R_{n, l_{j}^{-}}^{-} \quad\left(n \in \bar{N}_{-}\right)
$$

and

$$
A_{m}^{-}\left(\kappa_{n}^{+}-\kappa_{m}^{-}\right) T_{n m}^{-}=\sum_{j=1}^{M} C_{j}^{-} A_{l_{j}^{-}}^{-}\left(\kappa_{n}^{+}-\kappa_{l_{j}^{-}}^{-}\right) T_{n, l_{j}^{-}}^{-} \quad\left(n \in \bar{N}_{+}\right),
$$

where the $C_{j}^{-}$are determined from (3.16) reduced to

$$
\sum_{j=1}^{M} C_{j}^{-} A_{l_{j}^{-}}^{-} \tilde{R}_{l_{k}^{-}, l_{j}^{-}}^{-}=A_{m}^{-} \tilde{R}_{m, l_{k}^{-}}^{-} \quad(k=1,2, \ldots, M) .
$$

Here the embedding formulae (3.25) and (3.26) give the reflection/transmission coefficients $R_{n m}^{-}$and $T_{n m}^{-}$in terms of solely solutions forced by waves incident from $-\infty$. If instead $M_{-}=0$ and $M_{+}=M$ then the $C_{j}^{-}$and equations of (3.16) are omitted, and equations (3.13) and (3.17) become

$$
A_{m}^{-}\left(\kappa_{n}^{-}+\kappa_{m}^{-}\right) R_{n m}^{-}=\sum_{j=1}^{M} C_{j}^{+} A_{l_{j}^{+}}^{-}\left(\kappa_{n}^{-}-\kappa_{l_{j}^{+}}^{+}\right) T_{n, l_{j}^{+}}^{+} \quad\left(n \in \bar{N}_{-}\right)
$$

and

$$
A_{m}^{-}\left(\kappa_{n}^{+}-\kappa_{m}^{-}\right) T_{n m}^{-}=\sum_{j=1}^{M} C_{j}^{+} A_{l_{j}^{+}}^{-}\left(\kappa_{n}^{+}+\kappa_{l_{j}^{+}}^{+}\right) R_{n, l_{j}^{+}}^{+} \quad\left(n \in \bar{N}_{+}\right),
$$

where the $C_{j}^{+}$are now determined from (3.19) reduced to

$$
\sum_{j=1}^{M} C_{j}^{+} A_{l_{j}^{+}}^{+} \tilde{R}_{l_{k}^{+}, l_{j}^{+}}^{+}=-A_{m}^{-} \tilde{T}_{m, l_{k}^{+}}^{+} \quad(k=1,2, \ldots, M) .
$$

Now the embedding formulae (3.28) and (3.29) give the reflection/transmission coefficients $R_{n m}^{-}$and $T_{n m}^{-}$in terms of solely solutions forced by waves incident from $+\infty$.

An obvious variation follows upon replacing $H_{m}^{-} u_{m}^{-}$in (3.11) by $H_{m}^{+} u_{m}^{+}$, i.e., by instead seeking formulae for reflection/transmission coefficients for the solution forced by the $m$-th mode from $+\infty$, rather than $-\infty$. The appropriate adjustment to be made to equations (3.13), (3.17), (3.16) and (3.19) is simply that all superscripts + and - are interchanged. 


\section{3(c) Symmetry}

If the boundaries of the waveguide and the obstacles within it are arranged symmetrically about the line $x=0$ then $\bar{N}_{+}=\bar{N}_{-} \equiv \bar{N}$ (say), plus it is clear that $u_{m}^{ \pm}(x, y)=u_{m}^{\mp}(-x, y)$ and so

$$
R_{n m}^{-}=R_{n m}^{+}, T_{n m}^{-}=T_{n m}^{+} \quad(n, m \in \bar{N}) .
$$

In this case it is maybe not surprising to find that the number of solutions required for the embedding formulae can be reduced from $M$ to $M / 2=M^{\prime}$. First choose $M_{+}=M_{-}=$ $M / 2=M^{\prime}$, and then also $l_{1}^{ \pm}, l_{2}^{ \pm}, \ldots, l_{M_{ \pm}}^{ \pm}$equal to $l_{1}, l_{2}, \ldots, l_{M^{\prime}}$. Then the embedding formulae (3.13) and (3.17) take the form

$$
A_{m}^{-}\left(\kappa_{n}+\kappa_{m}\right) R_{n m}^{-}=\sum_{j=1}^{M^{\prime}} C_{j}^{-} A_{l_{j}}^{-}\left(\kappa_{n}+\kappa_{l_{j}}\right) R_{n, l_{j}}^{-}+\sum_{j=1}^{M^{\prime}} C_{j}^{+} A_{l_{j}}^{+}\left(\kappa_{n}-\kappa_{l_{j}}\right) T_{n, l_{j}}^{-}
$$

and

$$
A_{m}^{-}\left(\kappa_{n}-\kappa_{m}\right) T_{n m}^{-}=\sum_{j=1}^{M^{\prime}} C_{j}^{-} A_{l_{j}}^{-}\left(\kappa_{n}-\kappa_{l_{j}}\right) T_{n, l_{j}}^{-}+\sum_{j=1}^{M^{\prime}} C_{j}^{+} A_{l_{j}}^{+}\left(\kappa_{n}+\kappa_{l_{j}}\right) R_{n, l_{j}}^{-},
$$

where the constants $C_{j}^{ \pm}$are determined from (3.16) and (3.19) written as

$$
\sum_{j=1}^{M^{\prime}} C_{j}^{-} A_{l_{j}}^{-} \tilde{R}_{l_{k}, l_{j}}^{-}+\sum_{j=1}^{M^{\prime}} C_{j}^{+} A_{l_{j}}^{+} \tilde{T}_{l_{k}, l_{j}}^{-}=A_{m}^{-} \tilde{R}_{m, l_{k}}^{-} \quad\left(k=1,2, \ldots, M^{\prime}\right)
$$

and

$$
\sum_{j=1}^{M^{\prime}} C_{j}^{-} A_{l_{j}}^{-} \tilde{T}_{l_{k}, l_{j}}^{-}+\sum_{j=1}^{M^{\prime}} C_{j}^{+} A_{l_{j}}^{+} \tilde{R}_{l_{k}, l_{j}}^{-}=-A_{m}^{-} \tilde{T}_{m, l_{k}}^{-} \quad\left(k=1,2, \ldots, M^{\prime}\right) .
$$

Here the symmetry relations (3.31) have been used to ensure that all reflection and transmission coefficients rely only on the $M^{\prime}$ solutions $u_{l_{j}}^{-}\left(j=1,2, \ldots, M^{\prime}\right)$ for their calculation.

\section{Polygonal boundaries and obstacles}

In this section we consider the general case. Suppose that for $x \leq-L$ the waveguide occupies the region $a_{-}<y<b_{-}$, and for $x \geq L$ the waveguide occupies $a_{+}<y<b_{+}$, with $\left(a_{-}, b_{-}\right)$not necessarily equal to $\left(a_{+}, b_{+}\right)$. (A minor modification, which goes unpursued here, would see the boundaries for $x \geq L$, say, aligned at a rational angle to the $x$-axis).

For $-L<x<L$ the waveguide has boundaries which are piecewise linear and also contains polygonal obstacles. The parameter $M \geq 2$ counts the total number of corners present in the walls and obstacles. The precise position of the walls and obstacles is not needed for what follows, but what is required is that there exists a single positive integer $p$ for which the region in the waveguide exterior to the $j$-th corner (so inside the waveguide), for each $j=1,2, \ldots, M$, is of the form $r>0, s_{j} \pi / p<\theta<q_{j} \pi / p$, in which $s_{j}, q_{j} \in \mathbb{Z}$ with $q_{j}>s_{j}$ and $q_{j}-s_{j} \leq 2 p$, and where $(r, \theta)$ are local polar coordinates centred on the 
$j$-th corner with $\theta=0$ aligned in the positive $x$-direction. For example, the single strip scatterer example considered in section $3($ a) corresponds to the values $M=2$ and $p=1$, with $s_{1}=-1, q_{1}=1$ and $s_{2}=0, q_{2}=2$.

We introduce the differential operator $H=i \kappa^{-1} \partial_{x}$ and, in terms of which,

$$
H_{p, m}^{ \pm}=(-i \kappa)^{p}\left[T_{p}(H)-T_{p}\left( \pm \kappa_{m}^{ \pm} / \kappa\right) I\right]
$$

in which $T_{p}$ is the $p$-th Chebychev polynomial of the first kind. The operator $H_{p, m}^{ \pm}$is a straightforward modification of an operator introduced in (8) for use for scattering problems in $\mathbb{R}^{2}$, and an extension of the operator $H_{m}^{ \pm}$in $(3.10)$ ( since $H_{1, m}^{ \pm}=H_{m}^{ \pm}$). It clearly commutes with the Helmholtz operator, preserves the radiation conditions as $x \rightarrow \pm \infty$, and when applied to the solution $u_{m}^{ \pm}$annihilates the incident wave component $e^{\mp i \kappa_{m}^{ \pm} x} Y_{m}^{ \pm}(y)$ (since for any $\gamma, T_{p}(H) e^{i \gamma x}=T_{p}(-\gamma / \kappa) e^{i \gamma x}$ ). It is also designed to preserve boundary conditions (of Dirichlet, Neumann or constant impedance type) on surfaces inclined at angles $l \pi / p$ for $l \in \mathbb{Z}$. The details of the verification of this property are in (8), but, in brief, when applied to solutions of the Helmholtz equation (2.1) the operators $H=$ $i \kappa^{-1} \partial_{x}$ and $\hat{H}=i \kappa^{-1} \partial_{y}$ obey the same algebraic rules as cosine and sine, respectively, since $H^{2} u+\hat{H}^{2} u=-\kappa^{-2}\left(u_{x x}+u_{y y}\right)=-\kappa^{-2}\left(-\kappa^{2} u\right)=u$. So if we write $H \equiv \cos \tilde{\theta}$ and $\hat{H} \equiv \sin \tilde{\theta}$, and also introduce $H^{\prime}=i \kappa^{-1} \partial_{x^{\prime}}$, in which coordinates $\left(x^{\prime}, y^{\prime}\right)$ are oriented along and perpendicular to a boundary inclined at an angle $l \pi / p$ to the $x$-axis, respectively, we have

$$
\begin{aligned}
T_{p}\left(H^{\prime}\right) & =T_{p}\left(i \kappa^{-1}\left[\cos (l \pi / p) \partial_{x}+\sin (l \pi / p) \partial_{y}\right]\right)=T_{p}(\cos (l \pi / p-\tilde{\theta})) \\
& =\cos [p(l \pi / p-\tilde{\theta})]=(-1)^{l} \cos (p \tilde{\theta})=(-1)^{l} T_{p}(H) .
\end{aligned}
$$

Thus the operator $T_{p}(H)$ preserves Dirichlet, Neumann or constant impedance boundary conditions on any such boundary, since it can be written in terms of derivatives directed along that boundary. (An alternative proof in (11), for the Dirichlet case only but readily extendable to other boundary conditions, instead confirms that the operator $T_{p}(H)$ maps solutions of the Helmholtz equation for the appropriate local wedge problem to other such solutions, so that in particular the boundary conditions are preserved.) Finally, if $T_{p}(H)$ preserves the boundary conditions of interest then from (4.1) so too clearly does $H_{p, m}^{ \pm}$.

Now we determine the effect, in terms of overly-singular terms produced at the corners of the domain, of applying the operator $H_{p, m}^{-}$to the solution $u_{m}^{-}$(the case of $H_{p, m}^{+} u_{m}^{+}$is very similar). Sufficiently close to the $j$-th corner, for which $s_{j} \pi / p<\theta<q_{j} \pi / p$, the solution $u_{m}^{-}$ behaves like the solution of the corresponding Laplace equation, so has the form

$$
u_{m}^{-}=\sum_{n=1}^{Q} A_{n}^{(m, j)} r^{\nu_{n, j}} \sin \left[\nu_{n, j}\left(\theta-s_{j} \pi / p\right)\right]+O\left(r^{\nu_{Q+1, j}}\right), \quad \nu_{n, j}=n p /\left(q_{j}-s_{j}\right)
$$

in terms of local polar coordinates $(r, \theta)$ centred on the apex of the $j$-th corner, and for some constants $A_{n}^{(m, j)}$ and $Q \in \mathbb{N}$. Writing

$$
\psi_{k}=r^{\nu_{n, j}+k} \sin \left[\nu_{n, j}\left(\theta-s_{j} \pi / p\right)+k \theta\right],
$$

in terms of which the $n$-th term in (4.2) is $A_{n}^{(m, j)} \psi_{0}$, it is straightforward to show that

$$
H^{l} \psi_{k}=\left(i \kappa^{-1}\right)^{l}\left(\nu_{n, j}+k\right)\left(\nu_{n, j}+k-1\right) \ldots\left(\nu_{n, j}+k-l+1\right) \psi_{k-l} \quad\left(l \in \mathbb{N}_{0}\right) .
$$


Then using this and a standard representation of $T_{p}(H)$ (e.g. equation (22.3.6) in (14)) we have

$$
T_{p}(H) \psi_{0}=\frac{p}{2} \sum_{l=0}^{[p / 2]} \frac{(-1)^{l}(p-l-1) !}{l !(p-2 l) !} 2^{p-2 l} H^{p-2 l} \psi_{0} \equiv \sum_{l=0}^{[p / 2]} \Delta_{j, n, l} \psi_{-p+2 l},
$$

where

$$
\Delta_{j, n, l}=\frac{p(-1)^{l}(p-l-1) !}{2 l !(p-2 l) !} 2^{p-2 l}\left(i \kappa^{-1}\right)^{p-2 l} \nu_{n, j}\left(\nu_{n, j}-1\right) \ldots\left(\nu_{n, j}-p+2 l+1\right) .
$$

Here $\psi_{-p+2 l}=r^{\nu_{n, j}-p+2 l} \sin \left[\nu_{n, j}\left(\theta-s_{j} \pi / p\right)+(-p+2 l) \theta\right]$, and the overly-singular terms correspond to cases when the exponent of $r$ is negative, i.e. when $\nu_{n, j}-p+2 l<0$, or equivalently $l<\left(p-\nu_{n, j}\right) / 2$. Thus near the $j$-th corner

$$
T_{p}(H) u_{m}^{-} \sim \sum_{n=1}^{\left\langle q_{j}-s_{j}\right\rangle} A_{n}^{(m, j)} \sum_{l=0}^{\left\langle\left(p-\nu_{n, j}\right) / 2\right\rangle} \Delta_{j, n, l} r^{\nu_{n, j}-p+2 l} \sin \left[\nu_{n, j}\left(\theta-s_{j} \pi / p\right)+(-p+2 l) \theta\right],
$$

where now only the overly-singular terms are included in the summations. Here $\langle z\rangle=$ $\max \left\{n \in \mathbb{N}_{0}: n<z\right\}$ denotes the largest integer strictly smaller than $z$. Thus the total number of overly-singular terms, for all corners, is

$$
\hat{M}=\sum_{j=1}^{M} \sum_{n=1}^{\left\langle q_{j}-s_{j}\right\rangle} \sum_{l=0}^{\left\langle\left(p-\nu_{n, j}\right) / 2\right\rangle} 1=\sum_{j=1}^{M} \sum_{n=1}^{\left\langle q_{j}-s_{j}\right\rangle}\left(1+\left\langle\left(p-\nu_{n, j}\right) / 2\right\rangle\right) .
$$

Furthermore, because the differences between $T_{p}(H)$ and the operator $H_{p, m}^{-}$in (4.1) do not introduce additional overly-singular terms, the total number of overly-singular terms which arise after application of $H_{p, m}^{-}$to $u_{m}^{-}$is precisely $\hat{M}$.

The process described in section 3(b) can now be followed closely, the main changes being that the operator $H_{m}^{ \pm}$is replaced by $H_{p, m}^{ \pm}$, and $M, M_{+}, M_{-}$are replaced by $\hat{M}, \hat{M}_{+}, \hat{M}_{-}$ respectively. Equations (3.16) and (3.19) become

$$
(-1)^{p+1} \sum_{j=1}^{\hat{M}_{-}} C_{j}^{-} A_{l_{j}^{-}}^{-} \tilde{R}_{l_{k}^{-}, l_{j}^{-}}^{-}+\sum_{j=1}^{\hat{M}_{+}} C_{j}^{+} A_{l_{j}^{+}}^{+} \tilde{T}_{l_{k}^{-}, l_{j}^{+}}^{+}=A_{m}^{-} \tilde{R}_{m, l_{k}^{-}}^{-} \quad\left(k=1,2, \ldots, \hat{M}_{-}\right)
$$

and

$$
\sum_{j=1}^{\hat{M}_{-}} C_{j}^{-} A_{l_{j}^{-}}^{-} \tilde{T}_{l_{k}^{+}, l_{j}^{-}}^{-}+\sum_{j=1}^{\hat{M}_{+}} C_{j}^{+} A_{l_{j}^{+}}^{+} \tilde{R}_{l_{k}^{+}, l_{j}^{+}}^{+}=(-1)^{p} A_{m}^{-} \tilde{T}_{m, l_{k}^{+}}^{+} \quad\left(k=1,2, \ldots, \hat{M}_{+}\right)
$$

in which the definition of the scaled reflection and transmission coefficients in (3.8) and (3.15) is extended to

$$
\tilde{R}_{m j}^{ \pm}=\kappa \kappa_{m}^{ \pm}\left[T_{p}\left(\kappa_{j}^{ \pm} / \kappa\right)-T_{p}\left(-\kappa_{m}^{ \pm} / \kappa\right)\right] R_{m j}^{ \pm}, \quad \tilde{T}_{m j}^{ \pm}= \pm \kappa \kappa_{m}^{\mp}\left[T_{p}\left( \pm \kappa_{m}^{\mp} / \kappa\right)-T_{p}\left( \pm \kappa_{j}^{ \pm} / \kappa\right)\right] T_{m j}^{ \pm} .
$$


Here use has been made of the reciprocity relations

$$
\tilde{R}_{m j}^{ \pm}=(-1)^{p+1} \tilde{R}_{j m}^{ \pm}, \quad \tilde{T}_{m j}^{ \pm}=(-1)^{p} \tilde{T}_{j m}^{\mp} .
$$

If $p=1$, equations (4.4)-(4.7) reduce to their analogues in section $3(\mathrm{~b})$. With the $C_{j}^{ \pm}$ determined from (4.4) and (4.5), the embedding formulae themselves are

$$
A_{m}^{-} \tilde{R}_{n m}^{-}=\sum_{j=1}^{\hat{M}_{-}} C_{j}^{-} A_{l_{j}^{-}}^{-} \tilde{R}_{n, l_{j}^{-}}^{-}+(-1)^{p+1} \sum_{j=1}^{\hat{M}_{+}} C_{j}^{+} A_{l_{j}^{+}}^{+} \tilde{T}_{n, l_{j}^{+}}^{+}
$$

for $n \in \bar{N}_{-}$, and

$$
A_{m}^{-} \tilde{T}_{n m}^{-}=\sum_{j=1}^{\hat{M}_{-}} C_{j}^{-} A_{l_{j}^{-}}^{-} \tilde{T}_{n, l_{j}^{-}}^{-}+\sum_{j=1}^{\hat{M}_{+}} C_{j}^{+} A_{l_{j}^{+}}^{+} \tilde{R}_{n, l_{j}^{+}}^{+}
$$

for $n \in \bar{N}_{+}$. These formulae express $\tilde{R}_{n m}^{-}$and $\tilde{T}_{n m}^{-}$in terms of quantities which rely only on the solutions $u_{l_{j}^{ \pm}}^{ \pm}$for $j=1,2, \ldots, \hat{M}_{ \pm}$.

The corresponding embedding formulae for $\hat{R}_{n m}^{+}$and $\hat{T}_{n m}^{+}$are very similar, and given by

$$
A_{m}^{+} \tilde{R}_{n m}^{+}=\sum_{j=1}^{\hat{M}_{-}} C_{j}^{-} A_{l_{j}^{-}}^{-} \tilde{T}_{n, l_{j}^{-}}^{-}+\sum_{j=1}^{\hat{M}_{+}} C_{j}^{+} A_{l_{j}^{+}}^{+} \tilde{R}_{n, l_{j}^{+}}^{+}
$$

for $n \in \bar{N}_{+}$, and

$$
A_{m}^{+} \tilde{T}_{n m}^{+}=(-1)^{p+1} \sum_{j=1}^{\hat{M}_{-}} C_{j}^{-} A_{l_{j}^{-}}^{-} \tilde{R}_{n, l_{j}^{-}}^{-}+\sum_{j=1}^{\hat{M}_{+}} C_{j}^{+} A_{l_{j}^{+}}^{+} \tilde{T}_{n, l_{j}^{+}}^{+}
$$

for $n \in \bar{N}_{-}$. The coefficients $C_{j}^{ \pm}$are now determined by setting $n=l_{k}^{+}$in (4.10) and $n=l_{k}^{-}$in (4.11), using (4.7) to rewrite the left-hand sides in terms of quantities which do not depend on $u_{m}^{+}$, and solving the resulting equations. These formulae express $\tilde{R}_{n m}^{+}$and $\tilde{T}_{n m}^{+}$in terms of quantities which rely only on the solutions $u_{l_{j}^{ \pm}}^{ \pm}$for $j=1,2, \ldots, \hat{M}_{ \pm}$.

Note that if $p$ is even and $j=m$ then the coefficient multiplying $R_{m j}^{ \pm}$in (4.6) is zero, and so $R_{m m}^{ \pm}$cannot be recovered from $\tilde{R}_{m m}^{ \pm}$. Similarly, $T_{m m}^{ \pm}$cannot be recovered from $\tilde{T}_{m m}^{ \pm}$ if $\kappa_{m}^{+}=\kappa_{m}^{-}$, for any $p$. In such cases we divide the right-hand side of (4.8) (say) by the coefficient of $R_{n m}^{-}$in $\tilde{R}_{n m}^{-}$and then take the limit $n \rightarrow m$; use of L'Hôpital's rule yields an expression for $R_{m m}^{-}$in terms of the derivatives

$$
\left.\frac{\partial R_{n, l_{j}^{-}}^{-}}{\partial n}\right|_{n=m},\left.\quad \frac{\partial T_{n, l_{j}^{+}}^{+}}{\partial n}\right|_{n=m},
$$

quantities which again depend on $u_{l_{j}^{ \pm}}^{ \pm}$for $j=1,2, \ldots, \hat{M}_{ \pm}$. A similar limiting process has been used to recover scattering coefficients in special cases in other embedding problems (e.g. (15)). The details are not pursued here. 
Finally, if the waveguide and obstacles are symmetric about $x=0$ then the symmetry relations (3.31) become $\tilde{R}_{n m}^{ \pm}=\tilde{R}_{n m}^{\mp}, \tilde{T}_{n m}^{ \pm}=(-1)^{p+1} \tilde{T}_{n m}^{\mp}$. If $\hat{M}$ is even then we set $\hat{M}_{-}=$ $\hat{M}_{+}=\hat{M} / 2=\hat{M}^{\prime}$, say, and $l_{j}^{ \pm}=l_{j}$ for $j=1, \ldots, \hat{M}^{\prime}$; the embedding formulae (4.8),(4.9) become

$A_{m}^{-} \tilde{R}_{n m}^{-}=\sum_{j=1}^{\hat{M}^{\prime}} C_{j}^{-} A_{l_{j}}^{-} \tilde{R}_{n, l_{j}}^{-}+(-1)^{p+1} C_{j}^{+} A_{l_{j}}^{+} \tilde{T}_{n, l_{j}}^{+}, \quad A_{m}^{-} \tilde{T}_{n m}^{-}=\sum_{j=1}^{\hat{M}^{\prime}} C_{j}^{-} A_{l_{j}}^{-} \tilde{T}_{n, l_{j}}^{-}+C_{j}^{+} A_{l_{j}}^{+} \tilde{R}_{n, l_{j}}^{+}$,

where the $C_{j}^{ \pm}$are determined from (4.4), (4.5) modified to

$\sum_{j=1}^{\hat{M}^{\prime}}(-1)^{p+1} C_{j}^{-} A_{l_{j}}^{-} \tilde{R}_{l_{k}, l_{j}}^{-}+C_{j}^{+} A_{l_{j}}^{+} \tilde{T}_{l_{k}, l_{j}}^{+}=A_{m}^{-} \tilde{R}_{m, l_{k}}^{-}, \quad \sum_{j=1}^{\hat{M}^{\prime}} C_{j}^{-} A_{l_{j}}^{-} \tilde{T}_{l_{k}, l_{j}}^{-}+C_{j}^{+} A_{l_{j}}^{+} \tilde{R}_{l_{k}, l_{j}}^{+}=(-1)^{p} A_{m}^{-} \tilde{T}_{m, l_{k}}^{-}$

for $k=1, \ldots, \hat{M}^{\prime}$. If instead $\hat{M}$ is odd then minor adjustments yield formulae which rely only on $(1+\hat{M}) / 2$ solutions.

\section{4(a) Examples}

To illustrate the formulae, we consider some examples. In each case once the value of $\hat{M}$ is determined the embedding formulae are equations (4.8)-(4.11), or equation (4.12) if the waveguide is symmetric.

\section{4(a).1 A step-change in waveguide width}

Consider a waveguide with a step-change in width at $x=0$. The upper boundary is positioned at $y=0$; for $x<0$ the lower boundary is at $y=-b<0$, but for $x>0$ the lower boundary is at $y=-(b+a)<-b$. Thus the waveguide widens from width $b$ to width $a+b$ as the line $x=0$ is crossed left-to-right. Here $p=2$ and there are $M=2$ corners: at $(0,-b)$ with $s_{1}=-1, q_{1}=2$, and at $(0,-b-a)$ with $s_{2}=0, q_{2}=1$. The second corner is concave, with $\left\langle q_{2}-s_{2}\right\rangle=0$, so does not contribute overly-singular terms. For the first, $\left\langle q_{1}-s_{1}\right\rangle=2$ and $\nu_{n, 1}=2 n / 3$, so $\left\langle\left(p-\nu_{n, 1}\right) / 2\right\rangle=\langle(2-2 n / 3) / 2\rangle=\langle 1-n / 3\rangle=0$ for $n=1$, 2 , so that from (4.3), $\hat{M}=2$.

\section{4(a).2 A step-change in waveguide width, with a screen}

Consider the waveguide geometry of example 4(a).1, but with an additional thin straight screen extending from the corner of the step at $(0,-b)$ across the waveguide to $(0,-c)$, where $0<c<b$ (see fig. 2). Again $p=2$ but now there are $M=3$ corners: at $(0,-b)$ with $s_{1}=1, q_{1}=2$, at $(0,-b-a)$ with $s_{2}=0, q_{2}=1$, and at $(0,-c)$ with $s_{3}=-1, q_{3}=3$. The first and second corners are both concave; for the third, $\left\langle q_{3}-s_{3}\right\rangle=3$ and $\nu_{n, 3}=n / 2$, so from (4.3), $\hat{M}=\sum_{n=1}^{3} 1+\langle 1-n / 4\rangle=3$. The embedding formulae for this example are implemented in section 5 , below.

\section{4(a).3 A single barrier inclined at an angle $s \pi / p$ to the $x$-axis}

Here there are again $M=2$ corners in the domain, with $s_{1}=s-p$ and $q_{1}=s+p$, and $s_{2}=s-2 p$ and $q_{2}=s$, so that $q_{j}-s_{j}=2 p$ for $j=1,2$. Thus $\nu_{n, j}=n / 2$ for $j=1,2$, and 


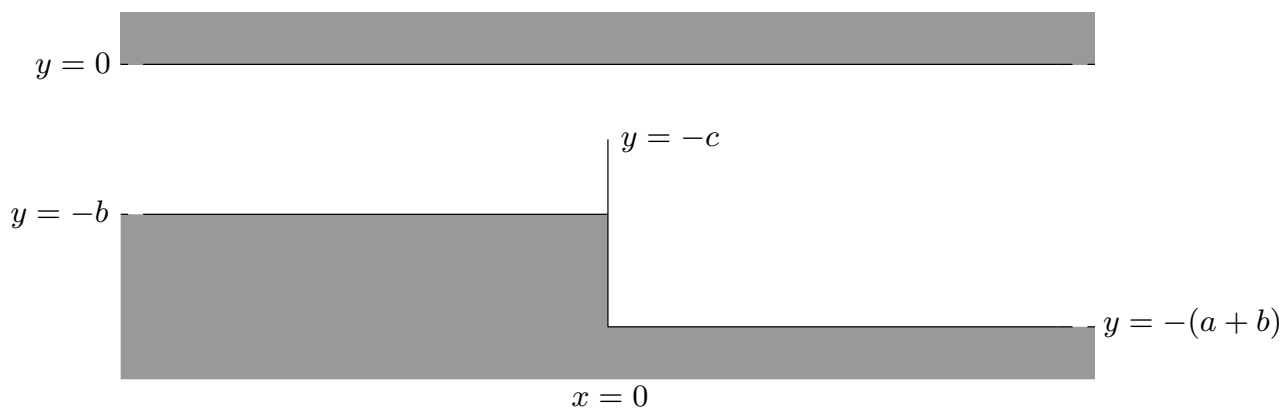

Fig. 2 Waveguide geometry for example 4(a).2.

so from (4.3)

$$
\hat{M}=\sum_{j=1}^{2} \sum_{n=1}^{\langle 2 p\rangle}(1+\langle(p-n / 2) / 2\rangle)=2(2 p-1)+2 \sum_{n=1}^{2 p-1}\langle p / 2-n / 4\rangle .
$$

If the barrier is aligned with the $x$-axis, as in section $3(\mathrm{a})$, then $p=1$ (and $s=0$ ) and $\hat{M}=2$, as expected.

\section{4(a).4 A single rectangular obstacle aligned with the $x$-axis}

In this case there $M=4$ corners, with $p=2$ and $\left(s_{1}, q_{1}\right)=(-1,2),\left(s_{2}, q_{2}\right)=(0,3)$, $\left(s_{3}, q_{3}\right)=(1,4)$ and $\left(s_{4}, q_{4}\right)=(-2,1)$. Then $q_{j}-s_{j}=3$ and $\nu_{n, j}=2 n / 3$ for $j=1,2,3,4$, giving

$$
\hat{M}=\sum_{j=1}^{4} \sum_{n=1}^{2}(1+\langle(2-2 n / 3) / 2\rangle)=4 \sum_{n=1}^{2}(1+\langle 1-n / 3\rangle)=4 \sum_{n=1}^{2} 1=8 .
$$

If the obstacle is positioned symmetric about $x=0$ then use of the symmetry properties reduces this figure to $\hat{M} / 2=4$.

\section{Numerical approximation for example 4(a).2}

For the case described in section 4(a).2 we provide an approximate numerical solution against which the embedding formula can be checked.

We focus on the solution of the problem for $u_{m}^{-}$; the approximation of $u_{m}^{+}$is very similar. In an extension of $(2.4), u_{m}^{-}$can be written as

$$
u_{m}^{-}(x, y)= \begin{cases}A_{m}^{-} e^{i \kappa_{m}^{-} x} Y_{m}^{-}(y)+\sum_{n=1}^{\infty} R_{n m}^{-} e^{-i \kappa_{n}^{-} x} Y_{n}^{-}(y) & (x<0) \\ A_{m}^{-} \sum_{n=1}^{\infty} T_{n m}^{-} e^{i \kappa_{n}^{+} x} Y_{n}^{+}(y) & (x>0)\end{cases}
$$

in which the $R_{n m}^{-}$and $T_{n m}^{-}$are coefficients to be determined. The solution is continuous 
above the step but equal to zero on the face of the step, so evaluating (5.1) at $x=0$ and using the orthogonality of the $Y_{n}^{ \pm}$yields the relations

$$
\left(\hat{u}_{m}^{-}, Y_{n}^{-}\right)=A_{m}^{-}\left(\delta_{n m}+R_{n m}^{-}\right), \quad\left(\hat{u}_{m}^{-}, Y_{n}^{+}\right)=A_{m}^{-} T_{n m}^{-} \quad(n \in \mathbb{N}),
$$

where $\hat{u}_{m}^{-}(y)=u_{m}^{-}\left(0^{ \pm}, y\right)$ for $-c<y<0, \delta_{n m}$ is the Kronecker delta, and the inner product notation $(u, v)=\int_{-c}^{0} u(y) \bar{v}(y) \mathrm{d} y$ has been used. The derivative $u_{m_{x}}^{-}$is also continuous across the step, so matching derivatives of $u_{m}^{-}$from (5.1), and using (5.2) to replace coefficients of evanescent modes, results in

$$
\begin{aligned}
\sum_{n=N_{-}+1}^{\infty} \kappa_{n}^{-}\left(\hat{u}_{m}^{-}, Y_{n}^{-}\right) Y_{n}^{-}(y) & +\sum_{n=N_{+}+1}^{\infty} \kappa_{n}^{+}\left(\hat{u}_{m}^{-}, Y_{n}^{+}\right) Y_{n}^{+}(y) \\
& =A_{m}^{-} \sum_{n=1}^{N_{-}} \kappa_{n}^{-}\left(\delta_{n m}-R_{n m}^{-}\right) Y_{n}^{-}(y)-A_{m}^{-} \sum_{n=1}^{N_{+}} \kappa_{n}^{+} T_{n m}^{-} Y_{n}^{+}(y)
\end{aligned}
$$

for $-c<y<0$. The solution of (5.3) is decomposed as

$$
\hat{u}_{m}^{-}(y)=A_{m}^{-} \sum_{n=1}^{N_{-}} \kappa_{n}^{-}\left(\delta_{n m}-R_{n m}^{-}\right) v_{n}^{-}(y)-A_{m}^{-} \sum_{n=1}^{N_{+}} \kappa_{n}^{+} T_{n m}^{-} v_{n}^{+}(y)
$$

where

$$
\sum_{n=N_{-}+1}^{\infty} \kappa_{n}^{-}\left(v_{p}^{ \pm}, Y_{n}^{-}\right) Y_{n}^{-}(y)+\sum_{n=N_{+}+1}^{\infty} \kappa_{n}^{+}\left(v_{p}^{ \pm}, Y_{n}^{+}\right) Y_{n}^{+}(y)=Y_{p}^{ \pm}(y)
$$

for $p \in \bar{N}_{ \pm}$, so that from (5.2) and (5.4),

$$
\left\{\begin{array}{l}
\sum_{p=1}^{N_{-}} \kappa_{p}^{-}\left(\delta_{p m}-R_{p m}^{-}\right)\left(v_{p}^{-}, Y_{n}^{-}\right)-\sum_{p=1}^{N_{+}} \kappa_{p}^{+} T_{p m}^{-}\left(v_{p}^{+}, Y_{n}^{-}\right)=\delta_{n m}+R_{n m}^{-} \quad\left(n, m \in \bar{N}_{-}\right) \\
\sum_{p=1}^{N_{-}} \kappa_{p}^{-}\left(\delta_{p m}-R_{p m}^{-}\right)\left(v_{p}^{-}, Y_{n}^{+}\right)-\sum_{p=1}^{N_{+}} \kappa_{p}^{+} T_{p m}^{-}\left(v_{p}^{+}, Y_{n}^{+}\right)=T_{n m}^{-} \quad\left(n \in \bar{N}_{+}, m \in \bar{N}_{-}\right) .
\end{array}\right.
$$

These can be written as

$$
F^{--} \kappa^{-}\left(I_{-}-R^{-}\right)-F^{+-} \kappa^{+} T^{-}=I_{-}+R^{-}, \quad F^{-+} \kappa^{-}\left(I_{-}-R^{-}\right)-F^{++} \kappa^{+} T^{-}=T^{-}
$$

in which $I_{ \pm}$is the $N_{ \pm} \times N_{ \pm}$identity matrix ( $I_{+}$is used below), $\kappa^{ \pm}=\operatorname{diag}\left\{\kappa_{1}^{ \pm}, \ldots, \kappa_{N_{ \pm}}^{ \pm}\right\}$,

$$
R^{-}=\left\{R_{n m}^{-}\right\}_{n, m \in \bar{N}_{-}}, \quad T^{-}=\left\{T_{n m}^{-}\right\}_{n \in \bar{N}_{+}, m \in \bar{N}_{-}},
$$

and

$$
\left\{\begin{array}{l}
F^{--}=\left\{\left(v_{p}^{-}, Y_{n}^{-}\right)\right\}_{n, p \in \bar{N}_{-}}, \quad F^{+-}=\left\{\left(v_{p}^{+}, Y_{n}^{-}\right)\right\}_{n \in \bar{N}_{-}, p \in \bar{N}_{+}} \\
F^{-+}=\left\{\left(v_{p}^{-}, Y_{n}^{+}\right)\right\}_{n \in \bar{N}_{+}, p \in \bar{N}_{-}}, \quad F^{++}=\left\{\left(v_{p}^{+}, Y_{n}^{+}\right)\right\}_{n, p \in \bar{N}_{+}}
\end{array}\right.
$$


A very similar procedure applied to the problem for $u_{q}^{+}$shows that (5.7) is complemented by

$$
-F^{--} \kappa^{-} T^{+}+F^{+-} \kappa^{+}\left(I_{+}-R^{-}\right)=T^{+}, \quad-F^{-+} \kappa^{-} T^{+}+F^{++} \kappa^{+}\left(I_{+}-R+\right)=I_{+}+R^{+}
$$

in which

$$
R^{+}=\left\{R_{n m}^{+}\right\}_{n, m \in \bar{N}_{+}}, \quad T^{+}=\left\{T_{n m}^{-}\right\}_{n \in \bar{N}_{-}, m \in \bar{N}_{+}} .
$$

Once approximations to the matrices in (5.8) are determined, (5.7) and (5.9) can be solved for $R^{ \pm}$and $T^{ \pm}$.

To numerically approximate the solutions to (5.5), we use the Galerkin method in conjunction with the Rayleigh-Ritz approximation

$$
v_{p}^{ \pm}(y) \approx \sum_{j=0}^{Q} \lambda_{p j}^{ \pm} \varphi_{j}(y)
$$

in which the $\lambda_{p j}^{ \pm}$are coefficients to be determined, the $\varphi_{j}$ are trial functions, and $Q \in \mathbb{N}_{0}$. If $\rho$ denotes the distance from the corner at $(0,-c)$ then we know that $u_{m}^{-} \propto \rho^{1 / 2}$ near $\rho=0$; also, $u_{m}^{-}=0$ at $y=0$. These conditions together motivate the choice

$$
\varphi_{j}(y)=\frac{\left(c^{2}-y^{2}\right)^{1 / 2} U_{2 j+1}(y / c)}{c \sqrt{2 b}(-1)^{j}(j+1)} \quad(j=0, \ldots, Q)
$$

in which $U_{j}$ is a Chebychev polynomial of the second kind, and the denominator in (5.11) is chosen to simplify later results. Note that $U_{2 j+1}(-y)=-U_{2 j+1}(y)$. Inserting (5.10) into (5.5) and then multiplying the result by $\varphi_{k}$ and integrating, results in the Galerkin equations

$$
\sum_{j=0}^{P} L_{k j} \lambda_{p j}^{ \pm}=\left(Y_{p}^{ \pm}, \varphi_{k}\right) \quad(k=0, \ldots, Q)
$$

where

$$
L_{k j}=\sum_{n=N_{-}+1}^{\infty} \kappa_{n}^{-}\left(\varphi_{j}, Y_{n}^{-}\right)\left(Y_{n}^{-}, \varphi_{k}\right)+\sum_{n=N_{+}+1}^{\infty} \kappa_{n}^{+}\left(\varphi_{j}, Y_{n}^{+}\right)\left(Y_{n}^{+}, \varphi_{k}\right) \quad(k, j=0, \ldots, Q) .
$$

Standard properties of Chebychev polynomials of the second kind show that

$$
\left(\varphi_{j}, Y_{n}^{-}\right)=(-1)^{n} n^{-1} J_{2 j+2}(n \pi c / b), \quad\left(\varphi_{j}, Y_{n}^{+}\right)=\sqrt{1+a / b}(-1)^{n} n^{-1} J_{2 j+2}\left(\frac{n \pi c}{a+b}\right)
$$

in which $J_{2 j+2}$ is a Bessel function of the first kind.

The terms in each series in (5.13) behave like $n^{-2}$ as $n \rightarrow \infty$. In detail,

$$
\kappa_{n}^{-}\left(\varphi_{j}, Y_{n}^{-}\right)\left(Y_{n}^{-}, \varphi_{k}\right)=a_{1} n^{-2}[1+\sin (2 n \pi c / b)]+a_{2} n^{-3} \cos (2 n \pi c / b)+O\left(n^{-4}\right)
$$

and

$$
\kappa_{n}^{-}\left(\varphi_{j}, Y_{n}^{+}\right)\left(Y_{n}^{+}, \varphi_{k}\right)=a_{3} n^{-2}[1+\sin (2 n \pi c /(a+b))]+a_{4} n^{-3} \cos (2 n \pi c /(a+b))+O\left(n^{-4}\right)
$$


where

$$
a_{1}=\frac{i(-1)^{j+k}}{c \pi}, \quad a_{2}=\frac{i(-1)^{j+k} b}{c^{2} \pi^{2}}\left(\frac{15}{4}+4(j+k)+2\left(j^{2}+k^{2}\right)\right),
$$

and $a_{3}=(1+a / b) a_{1}, a_{4}=(1+a / b)^{2} a_{2}$. Denote the terms in $n^{-2}$ and $n^{-3}$ on the righthand sides of (5.15) and (5.16) by $A_{n}^{-}$and $A_{n}^{+}$respectively. Then we can accelerate the convergence of the series in (5.13) by writing them as

$$
\sum_{n=N_{-}+1}^{\infty}\left[\kappa_{n}^{-}\left(\varphi_{j}, Y_{n}^{-}\right)\left(Y_{n}^{-}, \varphi_{k}\right)-A_{n}^{-}\right]+a_{1} \zeta(2)+\frac{a_{1}}{2 i}\left(\mu_{12}-\bar{\mu}_{12}\right)+\frac{a_{2}}{2}\left(\mu_{13}+\bar{\mu}_{13}\right)-\sum_{n=1}^{N_{-}} A_{n}^{-}
$$

and

$$
\sum_{n=N_{+}+1}^{\infty}\left[\kappa_{n}^{+}\left(\varphi_{j}, Y_{n}^{+}\right)\left(Y_{n}^{+}, \varphi_{k}\right)-A_{n}^{+}\right]+a_{3} \zeta(2)+\frac{a_{1}}{2 i}\left(\mu_{22}-\bar{\mu}_{22}\right)+\frac{a_{4}}{2}\left(\mu_{23}+\bar{\mu}_{23}\right)-\sum_{n=1}^{N_{+}} A_{n}^{+}
$$

respectively, in which $\zeta(2)=\pi^{2} / 6$, and $\mu_{1 l}=\operatorname{Li}_{l}\left(e^{2 i \pi c / b}\right)$ and $\mu_{2 l}=\operatorname{Li}_{l}\left(e^{2 i \pi c /(a+b)}\right)$ for $l=2,3$, where

$$
\operatorname{Li}_{l}(z)=\sum_{n=1}^{\infty} \frac{z^{n}}{n^{l}}=\frac{(-1)^{l-1}}{(l-2) !} \int_{0}^{1} t^{-1} \ln ^{l-2} t \ln (1-z t) \mathrm{d} t
$$

is the polylogarithm function (see (16), section 25.12), which can be evaluated accurately using standard quadrature techniques applied to its integral form. The terms in the remaining infinite series in (5.17) and (5.18) now each decay like $n^{-4}$, and in practice truncating each series at $n=10^{3}$ ensures that for all results displayed below values of $L_{k j}$ are accurate to at least 6 decimal places.

The right-hand side of (5.12) can be evaluated using (5.14); once the $\lambda_{p j}^{ \pm}$are determined from (5.12) approximations to the entries of the matrices in (5.8) follow using (5.10) and (5.14).

\section{5(a) Results}

To illustrate the efficiency of the solution technique and the use of the embedding formulae we set $a / b=2$ and $c / b=1 / 2$, so that the waveguide width in $x>0$ is double that in $x<0$, and the screen at $x=0$ extends halfway across the waveguide at $x=0^{-}$. We choose $\kappa b=13 \pi / 2$, for which $N_{-}=6$ and $N_{+}=19$.

The left-hand half of Table 1 (the columns labelled 'direct') displays values of a selection of reflection and transmission coefficients as $Q$ is increased, calculated directly using the approximation method described above. The convergence with increasing $Q$ is evidently very rapid; the convergence rate of other $R_{n m}^{ \pm}$and $T_{n m}^{ \pm}$is comparable. For smaller (larger) $\kappa_{b}$, smaller (larger) $Q$ is required in order to achieve the same accuracy. If the screen is small $(c / b \approx 1)$, larger values of $Q$ are required.

To implement the embedding formulae (4.8)-(4.11) we choose $\hat{M}_{-}=\hat{M}=3, \hat{M}_{+}=0$, and for simplicity set $l_{j}^{-}=j$ for $j=1,2,3$, so that the embedding formulae express reflection and transmission coefficients in terms of solutions forced by the first three incident modes from the left. The numerical scheme described above is used to determine approximations to these 


\begin{tabular}{|c|c|c|c|c|c|c|c|c|}
\hline \multirow[b]{2}{*}{$Q$} & \multicolumn{4}{|c|}{ direct } & \multicolumn{4}{|c|}{ embedding formula } \\
\hline & $\left|R_{54}^{-}\right|$ & $\left|T_{10,6}^{-}\right|$ & $\left|R_{11,12}^{+}\right|$ & $\left|T_{4,16}^{+}\right|$ & $\left|R_{54}^{-}\right|$ & $\left|T_{10,6}^{-}\right|$ & $\left|R_{11,12}^{+}\right|$ & $\left|T_{4,16}^{+}\right|$ \\
\hline 2 & 0.26063 & 0.03824 & 0.16697 & 0.06309 & $9 \times 10^{9}$ & $2 \times 10^{8}$ & $1 \times 10^{9}$ & $3 \times 10^{9}$ \\
\hline 3 & 0.31012 & 0.06376 & 0.16347 & 0.08833 & $4 \times 10^{7}$ & $1 \times 10^{6}$ & $5 \times 10^{6}$ & $2 \times 10^{7}$ \\
\hline 4 & 0.29684 & 0.06878 & 0.16405 & 0.08269 & $6 \times 10^{3}$ & $3 \times 10^{2}$ & $5 \times 10^{2}$ & $3 \times 10^{3}$ \\
\hline 5 & 0.29532 & 0.06920 & 0.16419 & 0.08188 & 0.29702 & 0.05134 & 0.18014 & 0.24485 \\
\hline 6 & 0.29527 & 0.06922 & 0.16420 & 0.08185 & 0.29524 & 0.06922 & 0.16420 & 0.08186 \\
\hline 7 & 0.29527 & 0.06922 & 0.16420 & 0.08185 & 0.29527 & 0.06922 & 0.16420 & 0.08185 \\
\hline
\end{tabular}

Table 1 Values of a selection of reflection and transmission coefficients, calculated directly and via the embedding formulae (4.8)-(4.11). Here $a / b=2, c / b=1 / 2$, and $\kappa b=13 \pi / 2$, so that $N_{-}=6$ and $N_{+}=19$. Details related to the implementation of the embedding formulae are described in the text.

three solutions; these are then inserted into equations (4.8)-(4.11) to yield approximations to all other reflection and transmission coefficients. The columns of Table 1 labelled 'embedding formula' display approximate $\left|R_{n m}^{ \pm}\right|$and $\left|T_{n m}^{ \pm}\right|$calculated in this way, for which the generating three solutions are calculated using the specified value of $Q$.

What is evident is that the embedding formulae can reproduce the values of $\left|R_{n m}^{ \pm}\right|$ and $\left|T_{n m}^{ \pm}\right|$which have been calculated directly, as they should, but the accuracy of the approximations calculated in this way depends critically on the accuracy of the approximation to the three generating solutions: inserting even only slightly (by $\sim 1 \%$ ) inaccurate approximations to the three generating solutions into the embedding formulae results in approximations to the other $\left|R_{n m}^{ \pm}\right|$and $\left|T_{n m}^{ \pm}\right|$which are many orders of magnitude in error. However, given the rapid convergence of the numerical scheme this requirement of sufficient accuracy in the approximations to the generating solutions is not prohibitive, and the change in magnitude of the values of coefficients calculated via the embedding formulae as the approximations to the generating solutions become more accurate is so marked that convergence could not be wrongly assumed too early. Conversely, it is clear that the satisfaction of the embedding formulae can be used to gauge the accuracy of the numerical results.

Interestingly, this behaviour is in contrast to that of identities linking $\left|R_{n m}^{ \pm}\right|$and $\left|T_{n m}^{ \pm}\right|$ representing conservation of energy, namely

$$
\kappa_{m}^{-}=\sum_{n=1}^{N_{-}} \kappa_{n}^{-}\left|R_{n m}^{-}\right|^{2}+\sum_{n=1}^{N_{+}} \kappa_{n}^{+}\left|T_{n m}^{-}\right|^{2}, \quad \kappa_{q}^{+}=\sum_{n=1}^{N_{-}} \kappa_{n}^{-}\left|T_{n q}^{+}\right|^{2}+\sum_{n=1}^{N_{+}} \kappa_{n}^{+}\left|R_{n q}^{+}\right|^{2},
$$

for $n \in \bar{N}_{-}, q \in \bar{N}_{+}$, which follow from setting $u=u_{m}^{-}, v=\bar{u}_{m}^{-}$, and $u=u_{q}^{+}, v=\bar{u}_{q}^{+}$in (2.6). These identities are satisfied to machine accuracy by approximate $\left|R_{n m}^{ \pm}\right|$and $\left|T_{n m}^{ \pm}\right|$ for all $Q \geq 0$, even when the approximations themselves are evidently inaccurate. Thus the numerical scheme automatically preserves the identities expressing conservation of energy, but does not preserve the embedding formulae. 


\section{Conclusions}

In this paper we extended the methods of (11), which itself built upon (8), to derive embedding formulae for wave propagation problems in two-dimensional waveguides whose boundaries are piecewise linear, and which contain obstacles whose boundaries are also piecewise linear. Within this class of waveguide geometry the only further restriction is that each linear portion of the waveguide or obstacle boundary must make an angle with the waveguide axis which is a rational multiple of $\pi$ (in radians).

Embedding formulae were constructed which expressed the reflection/transmission coefficients for all possible incoming modes in terms of reflection/transmission coefficients for any particular $\hat{M}$ solutions, with $\hat{M}$ is given in (4.3), and corresponds to the total number of overly-singular terms which arise by application of the particular differential operator $H_{p, m}^{ \pm}$ defined in (4.1). This operator is designed to both commute with the Helmholtz operator $\nabla^{2}+\kappa^{2} I$, and also preserve boundary conditions on all boundaries.

In section 5, the boundary-value problem for a particular example was formulated as an integral equation and solved approximately using Galerkin's method, and in particular approximations to the corresponding reflection and transmission coefficients were determined. The appropriate embedding formulae were then implemented, which gave accurate values for a range of reflection and transmission coefficients in terms of just (for this particular example) 3 particular solutions, provided the approximations to these particular solutions were sufficiently accurate.

The embedding formulae are evidently of most use in high-frequency problems for which the number of propagating modes to either side of the non-uniform waveguide region, $N_{ \pm}$, is large, and in particular when $N_{ \pm} \gg \hat{M}$. For low-frequency problems in complicated waveguides $\hat{M}$ may exceed $N_{ \pm}$, prohibiting use of the embedding formulae; equally, for highfrequency problems in relatively simple waveguides the relative benefit of the embedding formulae is high. If $\hat{M}>N_{ \pm}$an alternative route is to use generating solutions which are forced by evanescent waves rather than propagating waves, at least in the case of a uniform width waveguide containing polygonal obstacles. This extension is not pursued here.

Embedding formulae can be derived for a range of other similar situations with only minor modifications, the most obvious such example being the case for which the Dirichlet boundary condition is replaced by a Neumann condition throughout. Slightly more involved, though still perfectly tractable, is the case for which the boundary condition on each linear boundary section (of the waveguide or an obstacle) is either Neumann or Dirichlet or of constant impedance type, though the appropriate modification of the value of $\hat{M}$ for the latter case requires care. The boundary condition could also switch between these three types at a point where the boundary is straight, so long as the possibility of overly-singular terms arising at these points is considered.

Finally, only straightforward modifications of the method are required for waveguides which are bifurcated or trifurcated etc., or for the radiation problem in which a waveguide opens out into $\mathbb{R}^{2}$, provided all boundaries remain inclined at a rational multiple of $\pi$ to the $x$-axis. This final case would provide a link between the results presented in this paper and those of (11) related to scattering by polygons. 


\section{Acknowledgements}

I thank the two anonymous reviewers whose comments and suggestions helped to substantially improve the paper.

\section{References}

1. M.H. Williams, Diffraction by a finite strip, Q. J. Mech. Appl. Math. 35 (1982) 103-124.

2. D. Porter, K.-W.E. Chu, The solution of two wave-diffraction problems, J. Eng. Math. 20 (1986) 63-72.

3. N.R.T. Biggs, D. Porter, D.S.G. Stirling, Wave diffraction through a perforated breakwater, Q. J. Mech. Appl. Math. 53 (2000) 375-391.

4. N.R.T. Biggs, D. Porter, Wave diffraction through a perforated barrier of non-zero thickness, Q. J. Mech. Appl. Math. 54 (2001) 523-547.

5. N.R.T. Biggs, D. Porter, Wave scattering by a perforated duct, Q. J. Mech. Appl. Math. 55 (2002) 249-272.

6. C.M. Linton, Embedding formulas and singularities in acoustic scattering, in: Proceedings of IUTAM Symposium on Asymptotics, Singularities and Homogenisation in Problems of Mechanics, (2003), 15-22.

7. R.V. Craster, A.V. Shanin, E.M. Doubravsky, Embedding formulae in diffraction theory, Proc. R. Soc. Lond. A 459 (2003) 2475-2496.

8. R.V. Craster, A.V. Shanin, Embedding formulae for diffraction by rational wedge and angular geometries, Proc. R. Soc. Lond. A 461 (2005) 2227-2242.

9. E.A. Skelton, R.V. Craster, A.V. Shanin, V. Valyaev, Embedding formulae for scattering by three-dimensional structures, Wave Motion, 47 (2010) 299-317.

10. E.A. Skelton, R.V. Craster, A.V. Shanin, Embedding formulae for diffraction by nonparallel slits, Q. J. Mech. Appl. Math. 61 (2008) 93-116.

11. N.R.T. Biggs, A new family of embedding formulae for diffraction by wedges and polygons, Wave Motion, 43 (7) (2006) 517-528.

12. Y. Duan, W. Koch, C.M. Linton, M. McIver, Complex resonances and trapped modes in ducted domains, J. Fluid Mech., 571 (2007) 119-147.

13. Y. Duan, M. McIver, Embedded trapped modes near an indentation in an open channel, in: 17th International Workshop on Water Waves and Floating Bodies (eds. R.C.T. Rainey, S.F. Lee), (2002), Peterhouse, Cambridge, 37-40.

14. M. Abramowitz, I.A. Stegun, eds. Handbook of mathematical functions with formulas, graphs, and mathematical tables, New York: Dover Publications (1964).

15. C.A.J. Moran, N.R.T. Biggs, P.G. Chamberlain, Embedding formulae for wave diffraction by a circular arc, Wave Motion, to appear.

16. F.W.J. Olver, D.W. Lozier, R.F. Boisvert, C.W. Clark, eds. NIST Handbook of Mathematical Functions, Cambridge University Press (2010). 